\title{
A family of non-invertible prime links
}

\section{James M. McPherson}

The purpose of this paper is to show that there exists an infinite family of non-invertible prime 2-component links in $S^{3}$. It is then noted that the existence of such a family implies the existence of a family of non-invertible wild arcs in $S^{3}$, which are tame modulo an endpoint.

All our "knots" and "links" are oriented knots and links in $S^{3}$, which has its orientation fixed.

Let $Z_{1} \cup Z_{2}$ be a non-splittable 2-component link. By the main theorem of [1], $z_{1} \cup l_{2}$ has a unique factorisation into prime links, and by Theorem $I$ of the same paper, one and only one of the prime links involved is a 2-component link $\tau_{1}^{*} \cup Z_{?}^{*}$. (The other links are all l-component links, that is, knots.) We call $l_{1}^{*} u l_{2}^{*}$ the prime hub of $\tau_{1} \cup \tau_{2}$. Then we note the following

(a) $Z_{1} \cup l_{2}$ is F-isotopic (of. [4]) to its prime hub, and

(b) $\lambda\left(z_{1}^{*}, z_{2}^{*}\right)=\lambda\left(z_{1}, z_{2}\right)$, where $\lambda\left(z_{1}, z_{2}\right)$ denotes the linking number of $z_{1}$ with $z_{2}$.

Let $k_{1}$ be one of the non-invertible pretzel knots of Trotter [5], and let $k_{2}$ be an unknotted simple closed curve in $S^{3}-k_{1}$ which has linking number +1 with $k_{1}$. Then $k_{1} \cup k_{2}$ is a non-invertible link whose prime hub consists of two simply linked circles, and is therefore invertible.

The situation is not lost, however; it is still possible to construct non-invertible prime links from our "basic link" $k_{1} \cup k_{2}$. We proceed as follows.

Received 11 September 1970. 
Let $V$ be a closed regular neighbourhood of $k_{1}$ in $S^{3}-k_{2}$, whose orientation is chosen so that $\lambda\left(V, k_{2}\right)=+1$. Let $k_{1}(r)$ be a knot lying in the interior of $V$ with winding number $r>1$; that is, a meridian of $\mathrm{BdV}$ has linking number $r$ with $k_{1}(r)$. Denote by $L_{r}$ the non-splittable link $k_{1}(r) \cup k_{2}$. Because $\lambda\left(k_{1}(r), k_{2}\right)=r, L_{r}$ and $L_{s}$ represent different $F$-isotopy classes if $r \neq s$. The prime hubs $H_{r}$ and $H_{s}$ of $L_{r}$ and $L_{s}$ are therefore distinct if $r \neq s$. We will show that $L_{r}$ is non-invertible. Indeed, we will show that $L_{r}$ and $L_{r}^{-1}=k_{1}(r)^{-1} \cup k_{2}^{-1}=k_{1}(-r) \cup k_{2}^{-1}$ are not even $F$-isotopic.

If there is an F-isotopy from $L_{r}$ to $L_{r}^{-1}$, there exists a closed solid torus $V^{\prime} \subset s^{3}-k_{2}^{-1}$, and an orientation-preserving homeomorphism $h:\left(S^{3}, V\right) \rightarrow\left(S^{3}, V^{\prime}\right)$ such that

(i) $k_{\perp}(r)^{-1}$ has linking number $r$ with a meridian of $B d V^{\prime}$, and (ii) $k_{2}^{-1}$ has linking number +1 with a core of $V^{\prime}$. (ef. [4], Theorem 3.)

Now $V$ and $V^{\prime}$ have the same oriented "knot type" in $S^{3}$, that is their oriented longitudes are equivalently knotted. But statement (ii) implies that $h$ takes a longitude of $V$ to the inverse of a longitude of $V^{\prime}$, so that a longitude of $V$ represents an invertible knot type in $S^{3}$. Since an oriented longitude of $V$ has the same (oriented) type as the pretzel knot $k_{1}$, this is impossible. Thus $L_{r}$ is non-invertible.

If the prime hub $H_{r}=k_{1}(r) * \cup k_{2}^{*}$ is non-invertible, the family $\left\{H_{r}: r>1\right\}$ will satisfy our requirements.

Since $L_{r}$ and $H_{r}$ are $F$-isotopic, and $k_{1}(r)$ has winding number $r>1$ in $V$, we apply Theorem 3 of [4] to obtain an oriented closed solid torus $V^{*} \subset S^{3}-k_{2}^{*}$, which has linking number +1 with $k_{2}^{*}=k_{2}$, which contains $k_{1}(r)$ * in its interior with winding number $r$, and which 
is of the same oriented knot type as $V$. Replacing $k_{1}(r)$ by $k_{1}(r)$ * and $V$ by $V^{*}$ in the argument above, we see that $H_{r}$ and $H_{r}^{-1}$ are not $F$-isotopic, that is $H_{r}$ is non-invertible. This proves the result stated in the abstract.

APPLICATION. For $n>1$, it is known that there exists an uncountable family $A_{n}$ of non-invertible wild arcs which are tame modulo one endpoint, where they have penetration index $2 n+1$, (see [2], p. 91).

We can now complete this result by stating the following theorem for $n=1$. The proof will appear in [3].

THEOREM. There exists an uncountable family $A_{1}$ of non-invertible ares which are tome modulo one endpoint, at which they have penetration index three.

QUESTION. It has been shown by whitten [6] that there exist non-invertible two-component links in $S^{3}$ which have invertible components. Does there exist an infinite family of prime links with this property?

\section{References}

[1] Yoko Hashizume, "On the uniqueness of the decomposition of a link", Osaka Math. J. 10 (1958), 283-300.

[2] James M. McPherson, "Wild knots and arcs in a 3-manifold", Ph.D. thesis at the University of New South Wales, Kensington, 1970.

[3] James M. McPherson, "Wild arcs in 3-manifolds, i: Families of Fox-Artin arcs", submitted.

[4] N. Smythe, "Topological invariants of isotopy of links, I", Amer. J. Math. 92 (1970), 86-98.

[5] H.F. Trotter, "Non-invertible knots exist", Topology 2 (1964), 275-280. 
[6] W.C. Whitten, Jr, "A pair of non-invertible links", Duke Math. J. 36 (1969), 695-698.

School of General Studies,

Australian National University,

Canberra, ACT. 